\title{
The Implication of Dissipative Structure Theory to Enterprise Information System
}

\author{
Jinling $\mathrm{Li}$, Renjing Liu, and Shanshan Sheng \\ School of Management, Xi' an Jiaotong University,710049,Xi'an \\ Shaanxi,P.R.China $\square$ renjingl@163.com
}

\begin{abstract}
This paper uses the dissipative structure theory to generate fresh insights that may help enterprises better understand and manage the EIS, Because the EIS fundamental structure embodies many characteristics of dissipative system which balance 'chaos' with 'order', the paper analyzes EIS under the non-equilibrium management entropy model and points out the importance of introducing negative entropy into the EIS for the first time. Two suggestion of building extended EIS and component-based system was made for the future developments of EIS.
\end{abstract}

\section{Introduction}

Enterprise Information System is fundamentally a software system responsible for the organizational and technological integration of a firm (Al-Mashari [1]; Davenport [3]; Loonam and McDonagh [10]). They are also referred to as ERP, EWIS and ES. Davenport [3] believes that an EIS should be defined by its ability to "seamlessly" integrate business processes and information flows up and down. Most research works and commercial products, however, are lack of the architectural integrity and functional applicability to meet these sophisticated needs of enterprises (Choi, [9]).According research conducted by the Standish Group International shows that 40 percent of all EIS installations achieved only partial implementation; nearly 28 percent were scrapped as total failures; and only 25 percent were completed on time and within budget. None of the studies so far offer a systematic description of the core concepts that result in flexible and innovative systems. Furthermore, no broader analysis has so far been presented in the literature of the fundamental principles that lie behind EIS, as we have described them. This paper will draw upon the theory of dissipative structure and principles of entropy to identify the basic principles that define the underlying structure of EIS. This analysis will show that successful system is poised at a delicate balance between chaotic and order.

Please use the following format when citing this chapter: 


\section{Conceptual Background}

\subsection{The Dissipative Structure Theory and the Entropy Theory}

Nobel laureate Ilya Prigogine have described in detail how complex and selforganizing dynamic system is, which they designate 'dissipative structures' are generated from the behavior of 'chaotic' processes. Although Prigogine's model is based on chemical reactions, it generates profound insights into the 'new thermodynamics of open systems' that have broad applicability. It has been used to analysis the social and economic systems, issue like enterprise structure, corporation management etc. Certain conditions for a system to grow into a dissipative system are Open system, Far from equilibrium, nonlinear dynamic system.

Entropy, in general, refers to a measure of the amount of disorder in a system; high entropy means high disorder, while low entropy represents orderly status; thus, the higher entropy the more chaotic the system. Attaran and Zwick [6] define entropy as a 'measure ' of disorder, uncertainty, or homogeneity for analyzing many different phenomena. McClean [12] measured the continuous time entropy of labor stability in measuring the stability of a firm. Buchen and Kelly [7] applied the entropy concept to asset modeling under information theory. As can be seen in these cases, "a different definition is appropriate for different uses".

\subsection{The Enterprise Information System and its Character}

An effective EIS must be driven by organizational goals that can sustain the change of technologies over time. For an EIS, it is important to control the chaotic data in and out of the system. We can put the characteristic of EIS in the dissipative structure' way:

1) It is an open system: An open system has been defined (DOD/DISA) as a system that implements sufficient specifications for interfaces, and services to enable engineered applications software: to interoperate with other applications on local and remote systems; and to interact with users in a style that facilitates portability.

2) It is a system far from equilibrium: an EIS has many non-equilibrium factors, like the mismanagement of data; lack of safe protection; the lack of analysis on information and data; As Kauffman argues the future development can be obtained with clear level, sub-system and improved function

3) It is a nonlinear system: the information and data of EIS are from and for various different aspects and its operation is complicated and non-linear; some for facing the customer / supplier/inner sector, also there usually will be inner structure's revolution within the EIS to fit the need of the enterprise. 


\section{The Non-Equilibrium Management Entropy Model of EIS}

Basically there are many factors affect the EIS as the function of entropy to the system, the entropy in an EIS can be categorized into three kinds: the structure entropy, the operation entropy and the environment entropy.

Assume the total entropy for an EIS far from equilibrium is $S$, its structure entropy is $S_{1}$, operation entropy is $S_{2}$ and environment entropy is $S_{3}$. For Mathematic analyze, $S$ is the function of $S_{1}, S_{2}$ and $S_{3}$, as $S=f\left(S_{1}, S_{2}, S_{3}\right)$. Entropy is an extensive quantity, the entropy $S$ for an EIS far from equilibrium is the sum of the entropy $S_{i}$ for each part in the system, to wit $S=\int d S_{l}$. Use boolean algebra to depict the relationship between $S$ and $S_{i}$, then $S=S_{1}+S_{2}+S_{3}$.

At the mean time, the three kinds of entropy $S_{1}, S_{2}$ and $S_{3}$, can be subdivided into more specific factors, $S_{1}$ can be subdivided into EIS framework entropy $H_{1}$ and strategy entropy $\mathrm{H}_{2} ; \mathrm{S}_{2}$ can be subdivided into management entropy $\mathrm{H}_{3}$, manipulation entropy $H_{4}$, data analysis entropy, $H_{5}$, project entropy $H_{6} ; S_{3}$ can be divided into customer entropy $H_{7}$ and supplier entropy $H_{8}$, thus,

$$
\begin{aligned}
& S_{1}=H_{1}+H_{2}, S_{2}=\sum_{i=3}^{6} H_{i}, S_{3}=H_{7}+H_{8} \\
& H_{j}=-P_{1} \ln P_{1}-P_{2} \ln P_{2}-, \ldots-P_{n} \ln P_{n}=-\sum_{i=1}^{n} P_{i} \ln P_{i} \quad(j=1,2, \ldots, 7,8)
\end{aligned}
$$

Assume the evaluation variable of the entropy is $W$, we could set functionality $W_{1}$ as an evaluation variable of $S_{1}$, set usability $W_{2}$, flexibility $W_{3}$ as evaluation variables of $S_{2}$, set market reactivity $W_{4}$ as evaluation variable of $S_{3} . W$ can be weighed by degree of disorder $R$, then $R_{1}$ represents degree of function disorder, $R_{2}$ represents degree of usable disorder, $R_{3}$ represents degree of flexible disorder, $R_{4}$ represents degree of market reactive disorder. Let $H_{m}$ be the highest entropy for each kind of entropy, then $W=\sum_{i=1}^{4} W_{i}=-R, R=\sum_{i=1}^{4} R_{i}=1-\sum_{i=1}^{8} \frac{H_{i}}{H_{i m}}$

For the far from equilibrium system, the entropy change within time $d t$ is $d S=d_{e} S+d_{i} S \cdot d_{e} S$ is the entropy produced by exchanging with the environment, $d_{i} S$ is the entropy produced by internal consumption.

If in an EIS far from equilibrium, $d_{e} S<0, d_{i} S>0$ and $\left|d_{e} S\right|>d_{i} S$, we can make $d S=d_{e} S+d_{i} S<0$. Entropy is decreasing, dissipative system can be formed.

We can have the following conclusions: 1) For the EIS, the two kinds of key environment's input are: customer's data and supplier's data; 2) The internal 
activities in EIS make the entropy increase, and affect its order; 3) The environment's input help the system to survive and develop negative entropy.

\section{The Implications to EIS: Actively Introduce Negative Entropy}

\subsection{Extended the Scope of EIS System: Extended Enterprise}

To make EIS adapt quickly to external changes depends upon the degree to which it exhibits a clear external focus. Indicate by the model, the environment entropy customer entropy $S_{3}\left(H_{7}\right.$ and $\left.H_{8}\right)$ is the key entropy to the EIS. So the scope of EIS should be extended and make the system borderless. the customer entropy and supplier entropy act as the negative entropy to balance the chaos in the system. See Fig. 1.

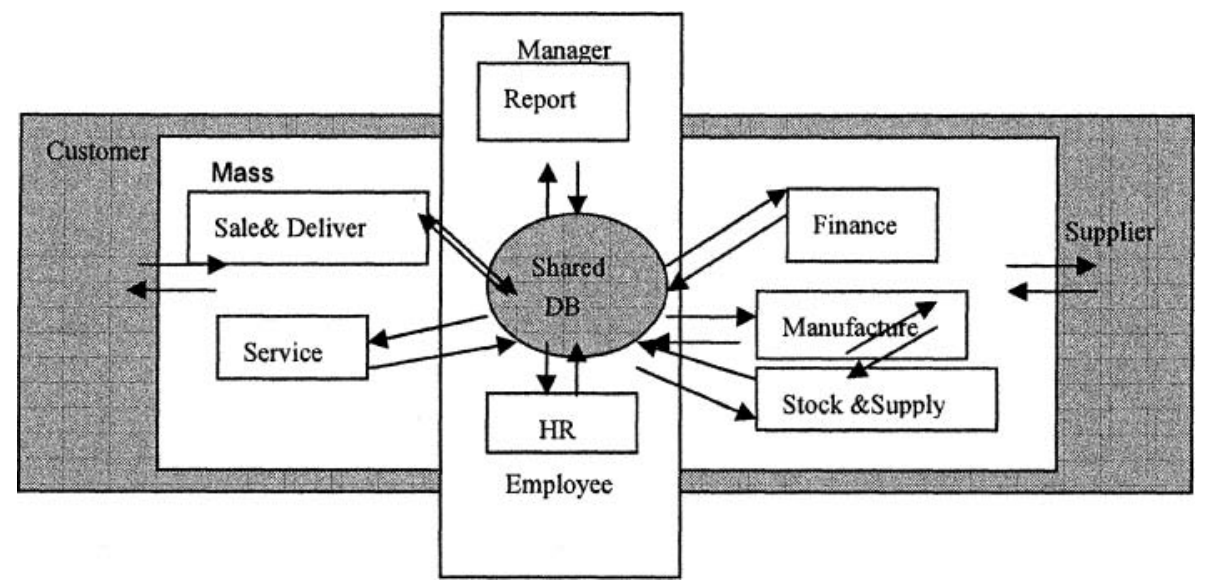

Fig. 1. Extended Enterpridse Information System

\subsection{Use the complication of the system: component-based system}

In an EIS, Two or more data are linked; their activities are so coordinated that when one data changes its state, the functions or activities of the other are affected. The EIS must be organized into flexible basic units that permit new structures to be identified, promote efficient exchanges of information. Let's see a case of changing in Bank EIS structure from traditional to component-based, which allows data of the innovative process to generate new 'orders.

\section{Conclusions and Future Research}

Ideas from the analysis of chaotic dynamic systems have been increasingly employed in economic analysis to describe non-linear, complex and creative organizational 
processes. This paper draws upon the dissipative structure theory lens to examine EIS for the first time. By using non-equilibrium management entropy model, the entropy statement of EIS is examined and the condition for an EIS to develop into dissipative system is found. EIS should be an extended system to actively introduce customer entropy and supplier entropy; component-based system can make use of the complication inside the system to balance 'chaos' with 'order'. The paper should be useful in contributing towards meeting enterprise mission in an efficient manner.

This EIS system consists of independent units tightly linked together and to external supplier. Since external suppliers in many cases provide inputs to other firms, and because they also have suppliers, this web of inter-industry effects will cause all markets to become increasingly chaotic. In the future research, this issue could be paid attention to.

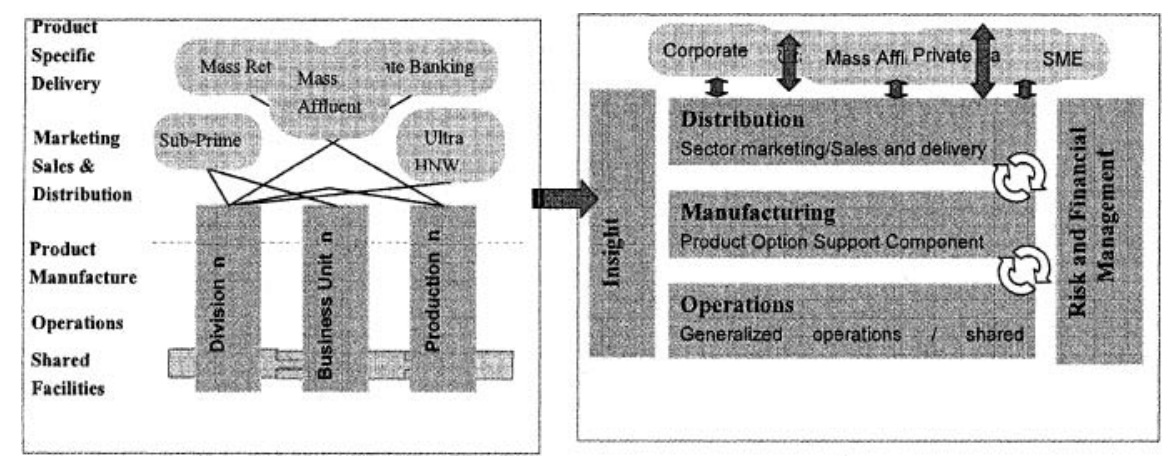

Fig. 2. Traditional 'monolithic' model and Component-based model

\section{Acknowledgements}

The authors would like to thank Professor Lida $\mathrm{Xu}$ for his insightful and helpful comments. We are grateful for the funding from Bureau of Science and Technology in Shaanxi Province (Grant No. 2004KR59), and "985" two term "07" base in Xian Jiaotong University. 


\section{References}

1. M. Al-Mashri, ERP: A Taxonomy of Critical Factors, European Journal of Operational Research 146(2), 352 (2003).

2. M. Arif, D. J. Kulonda, M. Proctor, and K. Williams, Before You Invest: An Illustrated Framework to Compare Conceptual Designs for an Enterprise Information System, Information Knowledge Systems Management 4119-135 (2004).

3. T.H. Davenport, Putting the Enterprise into the Enterprise System, Harvard Business Review 76(4), 121-31 (1998).

4. M. Hammer and J. Champy, Re-engineering the Corporation: A Manifesto for Business Evolution (Nicholas Brealcy, London. 1993)

5. B. Kaplan and D. Duchon, Combining Qualitative and Quantitative Methods in Information Systems Research: A Case Study, (1988)

6. M. Attaran and M. Zwick, An Information Theory Approach to Measuring Industrial Diversification Journal of Economic Studies 16, (1989).

7. P.W. Buchen and M. Kelly, The Maximum Entropy Distribution of an Asset Inferred from Option Prices, Journal of Financial and Quantitative Analysis 31 (1996).

8. C.S. Leem and S. Kim, Introduction to an Integrated Methodology for Development and Implementation of EIS, MIS Quarterly 11(2), (2001).

9. J.P. Choi, Information Concealment in the Theory of Vertical Integration, Journal of Economic Behavior \&Organization 35(1), 117 (1998).

10. J. Loonam and A. McDonagh, Exploring Top Management Support for the Introduction of EIS, Irish Journal of Management 26(1), 163-178 (2005).

11. R.A. Jenner, Technological Paradigms, Innovative Behavior and the Formation of Dissipative Enterprises, Small Business Economics 297-305 (1991).

12. S. McClean and T. Abodunde, Entropy as a Measure of Stability in a Manpower System, Journal of the Operational Research Society 29(9), 885-889 (1978). 